BNL - 73205-2004-CP

ATF-04C

\title{
Model Comparisons with STELIA Experimental Results
}

W. D. Kimura", M. Babzien ${ }^{\dagger}$, I. Ben-Zvi ${ }^{\dagger}$, L. C. Campbell ${ }^{*}$, D. B. Cline", C. E. Dilley*, J. C. Gallardo ${ }^{\dagger}$, S. C. Gottschalk*, K. P. Kusche ${ }^{* \dagger}$, R. H. Pantell ${ }^{\ddagger}$, I. V. Pogorelsky ${ }^{\dagger}$, D. C. Quimby ${ }^{*}$, J. Skaritka ${ }^{\dagger}$, L. C. Steinhauer ${ }^{8}$, V. Yakimenko ${ }^{\dagger}$, and F. Zhou

"STI Optronics, Inc., 2755 Northup Way, Bellevue, WA 98004

${ }^{\dagger}$ Brookhaven National Laboratory, Upton, NY 11973

'University of California, Los Angeles, Los Angeles, CA 90095

${ }^{\ddagger}$ Stanford University, Stanford, CA 94305

'University of Washington, Redmond Plasma Physics Laboratory, Redmond, WA 98052

September 2004

Presented at " $11^{\text {th }}$ Advanced Accelerator Concepts Workshop (AAC2004)", Stony Brook, New York, June $21-26,2004$. 


\section{DISCLAIMER}

This report was prepared as an account of work sponsored by an agency of the United States Government. Neither the United States Government nor any agency thereof, nor any of their employees, nor any of their contractors, subcontractors or their employees, makes any warranty, express or implied, or assumes any legal liability or responsibility for the accuracy, completeness, or any third party's use or the results of such use of any information, apparatus, product, or process disclosed, or represents that its use would not infringe privately owned rights. Reference herein to any specific commercial product, process, or service by trade name, trademark, manufacturer, or otherwise, does not necessarily constitute or imply its endorsement, recommendation, or favoring by the United States Government or any agency thereof or its contractors or subcontractors. The views and opinions of authors expressed herein do not necessarily state or reflect those of the United States Government or any agency thereof. 


\title{
Model Comparisons with STELLA Experimental Results
}

\author{
W. D. Kimura*, M. Babzien ${ }^{\dagger}$, I. Ben-Zvi , L. C. Campbel1", D. B. Cline", \\ C. E. Dilley*, J. C. Gallardo ${ }^{\dagger}$, S. C. Gottschalk*, K. P. Kusche* \\ R. H. Pantell ${ }^{\ddagger}$, I. V. Pogorelsky ${ }^{\dagger}$, D. C. Quimby ${ }^{*}$, J. Skaritka ${ }^{\dagger}$, \\ L. C. Steinhauer ${ }^{8}$, V. Yakimenko ${ }^{\dagger}$, and F. Zhou \\ "STI Optronics, Inc., 2755 Northup Way, Bellevue, WA 98004 \\ ${ }^{\dagger}$ Brookhaven National Laboratory, Upton, NY 11973 \\ "University of California, Los Angeles, Los Angeles, CA 90095 \\ ${ }^{\ddagger}$ Stanford University, Stanford, CA 94305 \\ ${ }^{\S}$ University of Washington, Redmond Plasma Physics Laboratory, Redmond, WA 98052
}

\begin{abstract}
High-trapping efficiency and narrow energy spread in a staged laser acceleration system was demonstrated during the Staged Electron Laser Acceleration (STELLA) experiment. The experiment used inverse free electron lasers (IFEL) driven by the Brookhaven National Laboratory Accelerator Test Facility (ATF) $\mathrm{CO}_{2}$ laser. The 1st IFEL modulated the electron beam energy. A subsequent chicane created a train of $\sim 3$ fs-long microbunches separated by 10.6 microns. These microbunches are trapped and accelerated in a 2nd IFEL where up to $80 \%$ trapping efficiencies and energy spreads down to $0.36 \%(1-\sigma)$ were measured. This paper presents additional model comparisons with the data, and discusses the strengths and limitations of the model.
\end{abstract}

\section{INTRODUCTION}

The Staged Electron Laser Acceleration (STELLA) experiment concluded with a successful demonstration of high trapping efficiency and narrow energy spread ("monoenergetic"). Details of the experiment can be found elsewhere [1] as well as the precursor experiment where staging between two laser-driven acceleration devices was first demonstrated [2]-[3].

Figure 1 shows a schematic of the STELLA experiment. Briefly, the STELLA experiment consisted of an inverse free electron laser (IFEL) [4] buncher (IFEL1) that imparts $\mathrm{a} \approx \pm 0.5 \%$ peak-to-peak energy modulation on a $45-\mathrm{MeV} e$-beam from the microwave-driven linac at the Brookhaven National Laboratory Accelerator Test Facility (ATF). This modulated $e$-beam travels through a hybrid permanent-magnet (PM)/electromagnet (EM) chicane. The PM part of the chicane is designed to cause the electrons to form microbunches at the output of the chicane. The EM part is used 


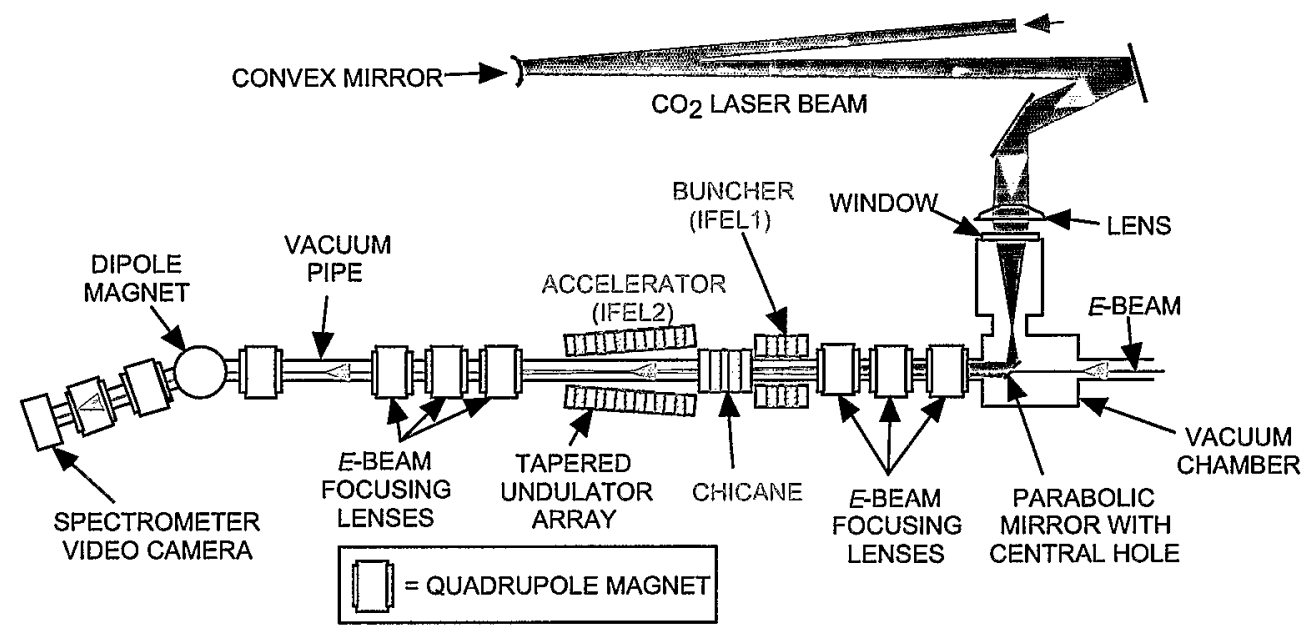

FIGURE 1. Schematic layout for the STELLA experiment.

to adjust the arrival time of the microbunches with respect to the laser field in the 2nd IFEL (IFEL2). This 2nd IFEL features a 12\% energy-tapered wiggler and is used to trap and accelerate the microbunches. The measured magnetic field of IFEL2 is given in Fig. 2. Partial strength magnets at the ends of the wiggler are designed to cause zero net deflection of the $e$-beam traversing the wiggler. A single high-power laser beam from the ATF $\mathrm{CO}_{2}$ laser $\left(\lambda_{\mathrm{L}}=10.6 \mu \mathrm{m}\right)$ drives both IFEL1 and IFEL2. The $e$ beam energy spectrum is measured using a spectrometer with an energy acceptance of $\pm 20 \%$ and a measured resolution of $0.14 \%(1 \sigma)$.

Besides demonstrating the important capabilities of staging, trapping, and monoenergetic acceleration, which are needed by a practical laser-driven linear accelerator ("laser linac"), another aim of this program was to provide data to validate the computer models. These models are versatile tools that can be used to develop designs for advanced IFEL systems, such as a 1-GeV IFEL laser linac [5].

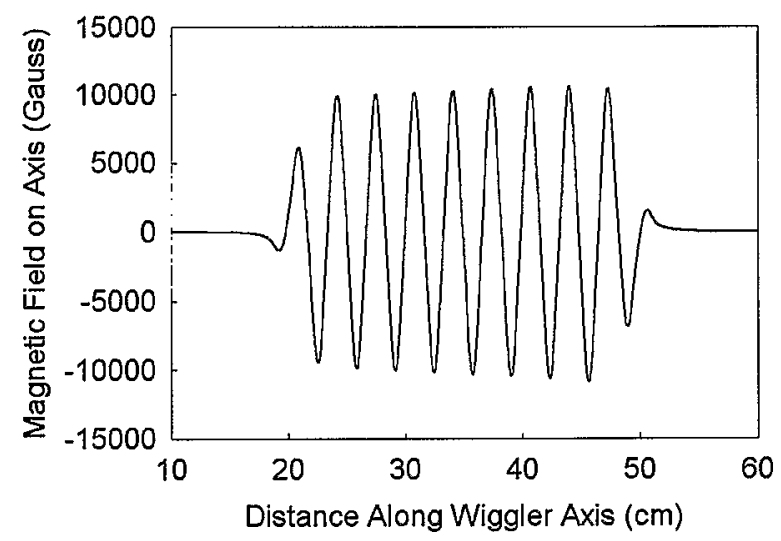

FIGURE 2. Measured magnetic field of IFEL2 wiggler. 
This paper compares the model predictions with the experimental data in more detail and discusses how well the model is able to predict the results.

\section{STELLA IFEL MODEL}

The STELLA IFEL model has been described elsewhere [2], [6]. The primary engine for the model is an IFEL module that uses the FEL equations [7] to solve for the electron/laser-field interactions along the length of the wiggler for an ensemble of individual electrons (typically 5,000 electrons distributed initially uniformly over phase). The complete $e$-beam characteristics are modeled including emittance and initial energy spread. Focusing of the $e$-beam and laser beam within the module can be defined. It is also possible to have less than perfect spatial and angular overlap of the beams within the wiggler. The program can use the actual measured magnetic field distribution of the wiggler or it can use a predefined distribution. A simple means for approximating the effects of space-charge is also available. Separate analysis and modeling [8] indicated that space-charge and coherent synchrotron radiation were not significant during the experiment and, hence, could be ignored in the simulations.

The IFEL module produces a file containing each electron's final transverse position $(x, y)$, trajectory angle $\left(\theta_{\mathrm{x}}, \theta_{\mathrm{y}}\right)$, starting and ending phase, and energy $(E)$. From these parameters one can plot various dependences such as the number of electrons versus final energy (i.e., energy spectrum), energy versus phase (i.e., phasespace plot), and longitudinal density distribution (i.e., bunch profile). The IFEL modules can be staged where each module takes the output file information from the previous module and uses it as the initial electron condition.

\section{COMPARISON OF DATA AND MODEL PREDICTIONS}

Extensive comparisons with the STELLA data and model have been presented elsewhere [1], [6]. This paper will concentrate on more in-depth comparisons of one particular energy spectrum. Figure 3 gives the measured spectrum and model predictions for a case not shown in the other publications. In fact, the trapping efficiency $(\approx 40 \%)$ and energy spread of the accelerated microbunch $[0.77 \%(1 \sigma)]$ is in between the values for the examples shown previously.

We see in Fig. 3(a) very good agreement between the model and data for the energy spectrum of the accelerated trapped electrons. However, there is still some disagreement between the model and data for the electrons near zero energy shift. This will be discussed later.

Table 1 lists the major model parameters used in the simulation shown in Fig. 3. The Rayleigh range $Z_{\mathrm{R}}$ in the model is calculated based upon the focusing optics used during the experiment, i.e., the convex mirror, focusing lens, and parabolic mirror shown in Fig. 1. Based upon the measured laser beam waist in the center of the IFEL2 wiggler $\left[\approx 1.5 \mathrm{~mm}\right.$ (full-width-at-half-maximum)], the effective value of $Z_{\mathrm{R}}$ during the 


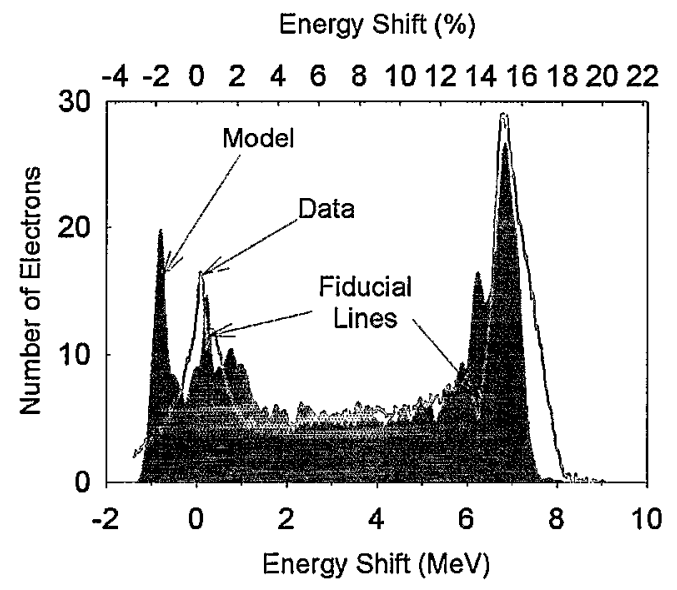

(a)

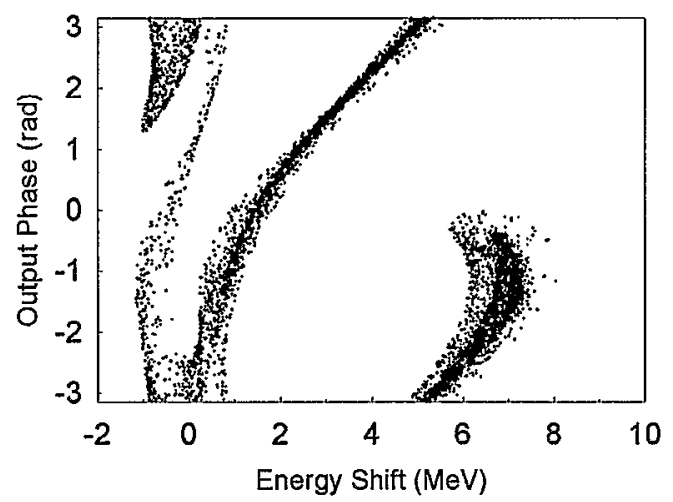

(b)

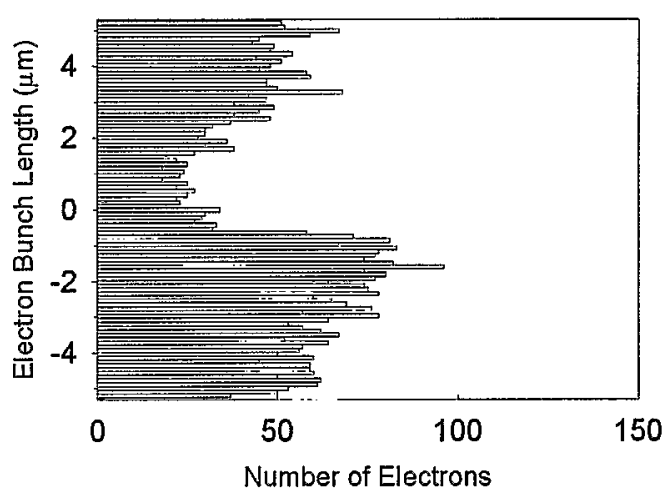

(c)

FIGURE 3. Sample results from STELLA experiment. (a) Electron energy spectrum of data (line) and model (histogram). (b) Energy-phase plot from model. Projection of this plot onto the energy axis gives (a). (c) Electron longitudinal distribution. Projection of the energy-phase plot (b) onto the phase axis gives (c).

experiment was over twice the value used in the model. This is not surprising since the laser beam is not diffraction limited and apertures along the laser beam transport line introduce diffraction effects. For $Z_{R}=21.3 \mathrm{~cm}$, the laser intensity at the center of the IFEL2 wiggler in the model is $1.1 \mathrm{TW} / \mathrm{cm}^{2}$. We estimate the laser intensity in IFEL2 during the experiment ranged from $1.2-1.9 \mathrm{TW} / \mathrm{cm}^{2}$, where the range of intensities is caused by an uncertainty in the amount of laser energy in the central lobe of the Airy-pattern focus at the center of the wiggler.

Because the amount of energy gain in a tapered wiggler is set by the amount of taper and not by the amount of laser power, as long as the laser intensity is above the minimum needed to drive the tapered wiggler, the peak energy is independent of the laser intensity. What does change as the laser intensity increases above the minimum drive intensity is that the trapped electrons begin to make synchrotron oscillations 
TABLE 1. Major model parameters used to generate results shown in Fig. 1.

\begin{tabular}{lcc}
\hline \multicolumn{1}{c}{ Parameter } & Value & Comments \\
\hline$E$-beam energy & $45 \mathrm{MeV}$ & Same as experiment. \\
$E$-beam $\Delta E / E(1 \sigma)$ & $0.03 \% \mathrm{x}$ & Same as experiment. \\
$E$-beam normalized emittance & $1.5 \mathrm{~mm}-\mathrm{mrad}$ & Same as experiment. \\
$E$-beam transverse offset & $0.05 \mathrm{~cm}$ & In $x$-direction only, no offset in $y$, see text. \\
Laser wavelength & $10.6 \mu \mathrm{m}$ & Same as experiment. \\
Laser Rayleigh range & $21.3 \mathrm{~cm}$ & See text for further explanation. \\
Laser intensity in IFEL2 & $1.1 \mathrm{TW} / \mathrm{cm}^{2}$ & Based on Rayleigh range, see text. \\
\hline
\end{tabular}

within the energy-phase ellipse. The group of accelerated electrons seen in the lower right of Fig. 3(b) are just beginning to turn and make this first synchrotron oscillation.

Therefore, the slight difference in the laser intensity used in the model and measured during the experiment primarily affects the energy spread of the accelerated electrons. However, we see there are only minor differences in the widths indicating that this feature of the energy spectrum is not strongly dependent on the laser intensity.

A more prominent feature of the spectrum is the electrons distributed between zero energy gain and the accelerated electrons. As mentioned in the previous publications [1], we believe this is caused by the laser beam and $e$-beam being slightly offset from each other inside the IFEL2 wiggler. Evidence for this occurring can be seen in Fig. 4, which the same data as in Fig. 3 except the $0.05-\mathrm{cm}$ transverse offset in the model (see Table 1) has been set to zero, i.e., no offset. We see most of the electrons in the middle of the spectrum have moved away and apparently a large number of them are now in the accelerated peak of electrons. This would be expected since with zero offset the electrons in the middle of the spectrum should experience the full acceleration.

Figure 3(c) shows the electron microbunch longitudinal profile. The aforementioned offset between the $e$-beam and laser beam appears to cause loss of many of the electrons in the microbunch, thereby, leading to a shrunken microbunch profile. This situation is greatly improved if the offset is removed [see Fig. 4(c)].

Returning to the difference between the data and model near zero energy shift, from the energy-phase plot [Fig. 3(b)], the electrons near zero energy shift originate from a wide and convoluted phase distribution. This accounts for the multiple peaks seen in the model simulation. The data on the other hand displays a single peak of electrons at zero energy shift.

Attempts were made to adjust the model parameters to cause a single peak at zero energy shift while preserving good agreement with the rest of the spectrum, i.e., the accelerated peak of trapped electrons. One parameter that can vary during the experiment is the peak magnetic field of the IFEL2 wiggler due to temperature changes in the ATF Experimental Hall. This will change the resonance condition for the tapered wiggler. Changing this peak value in the model does shift the energyphase plot in phase and can create an approximate single peak near zero energy shift. However, because the resonance condition is no longer well satisfied, the peak energy gain also decreases in disagreement with the data. Changing the e-beam energy slightly can re-establish this peak gain, but in doing so it also reshifts the energy-phase 


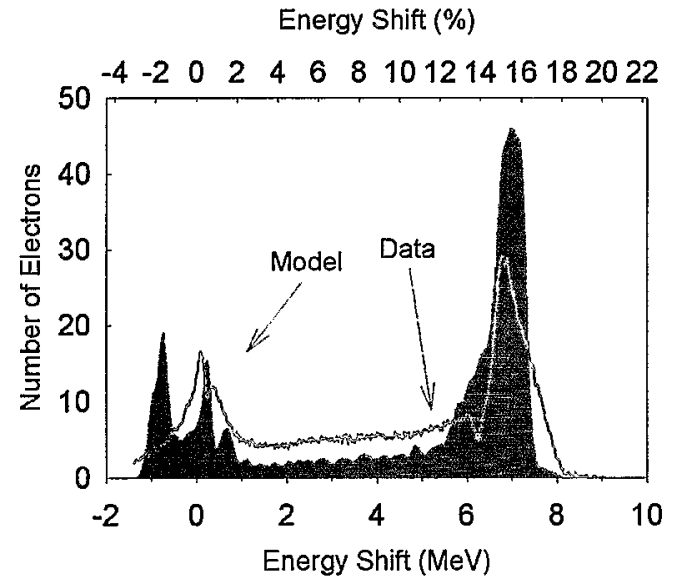

(a)

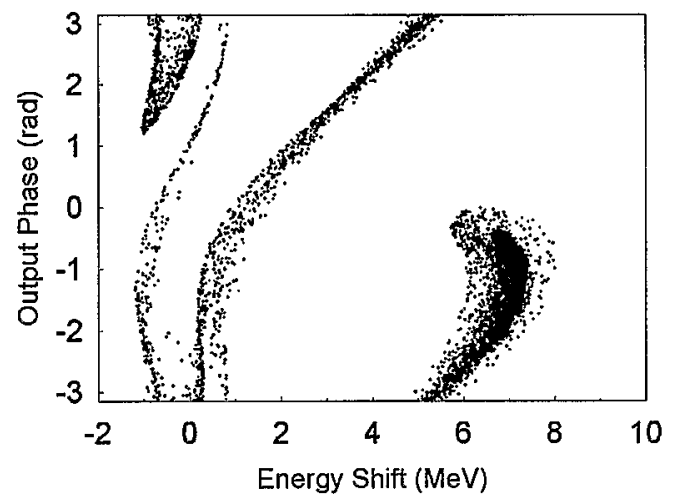

(b)

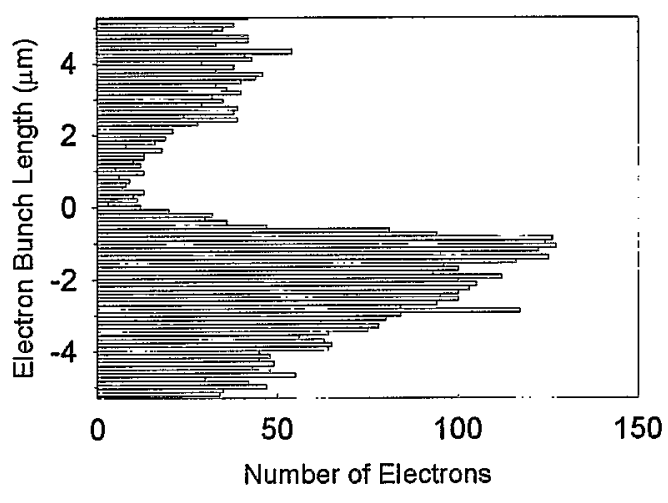

(c)

FIGURE 4. Energy spectrum for the same data as shown in Fig. 3, but with zero offset between the $e$ beam and laser beam in the model simulation.

plot in phase back to its original position. This makes the single peak near zero energy shift disappear and the multiple peaks reappear.

Hence, the cause of the single peak near zero energy shift is still not well understood.

\section{CONCLUSIONS}

Comparisons between the STELLA model and data reveal much information about the electron dynamics occurring during the IFEL interaction. In general the model gives accurate predictions on the overall performance of the individual IFEL devices and a system of devices. However, caution is warranted when attempting to derive finer details from the electron energy spectra. This spectra represents integrated data in more than one dimension. It is integrated over phase, over spatial dimensions and angles, and over time. And while the model is capable of separating these 
dependences, the results it yields are not necessarily unique in nature. Furthermore, a lack of knowledge of all the system parameters, for example, the exact trajectory of the $e$-beam through the wiggler magnetic field, limits the accuracy of the model.

Nevertheless, the model is a powerful tool that can be used to confidently design and predict the basic performance of IFELs and IFEL systems.

\section{ACKNOWLEDGMENTS}

This work was supported by the U.S. Department of Energy, Grant Nos. DE-FG0398ER41061, DE-AC02-98CH10886, and DE-FG03-92ER40695.

\section{REFERENCES}

1. W. D. Kimura, et al., Phys. Rev. Lett. 92, 054801 (2004).

2. W. D. Kimura, et al., Phys. Rev. ST Accel. Beams 4, 101301 (2001).

3. W. D. Kimura, et al., Phys. Rev. Lett. 86, 4041-4043 (2001).

4. R. B. Palmer, J. Appl. Phys. 43, 3014 (1972).

5. W. D. Kimura, "Conceptual Design for a 1-GeV IFEL Accelerator," in these Proceedings.

6. W. D. Kimura, et al., "Detailed Experimental Results for High-Trapping Efficiency and Narrow Energy Spread in a Laser-Driven Accelerator," submitted for publication in Phys. Rev. Spec. Topics - Accel. Beams, 2004.

7. N.M. Kroll, P.L. Morton, and M.N. Rosenbluth, IEEE J. Quant. Electron. QE-17, 1436 (1981).

8. F. Zhou, D. B. Cline, and W. D. Kimura, Phys. Rev. ST Accel. Beams 6, 054201 (2003). 